\title{
Disappearing Pulses in Vela X-1
}

\author{
P. Kretschmar ${ }^{1,2}$, I. Kreykenbohm ${ }^{2}$, J. Wilms ${ }^{2}$, R. Staubert ${ }^{2}$, \\ W. A. Heindl ${ }^{3}$, D. E. Gruber ${ }^{3}$, R. E. Rothschild ${ }^{3}$ \\ ${ }^{1}$ INTEGRAL Science Data Centre, Ch. d'Ecogia 16, 1290 Versoix, Switzerland \\ ${ }^{2}$ Institut für Astronomie und Astrophysik - Astronomie, Waldhäuser Str. 64, D-72076 Tübingen, Germany \\ ${ }^{3}$ CASS, University of California at San Diego, La Jolla, CA 92093, U.S.A.
}

\begin{abstract}
We present results from a $20 \mathrm{~h}$ RXTE observation of Vela X-1, including a peculiar low state of a few hours duration, during which the pulsation of the X-ray emission ceased, while significant non-pulsed emission remained. This "quiescent state" was preceded by a "normal state" without any unusual signs and followed by a "high state" of several hours of increased activity with strong, flaring pulsations. While there is clear spectral evolution from the normal state to the low state, the spectra of the following high state are surprisingly similar to those of the low state.
\end{abstract}

\section{INTRODUCTION}

Vela X-1 (4U 0900-40) is an eclipsing high mass X-ray binary consisting of the $23 M_{\odot}$ B0.5Ib supergiant HD 77581 and a neutron star with an orbital period of $8.964 \mathrm{~d}$ and a spin period of about $283 \mathrm{~s}$ (van Kerkwijk et al., 1995, and references therein). The persistent X-ray flux from the neutron star is known to be very variable exhibiting strong flares and low states. Inoue et al. (1984) and Kreykenbohm et al. (1999) have observed low states of near quiescence where no pulsations were seen for a short amount of time. Before or after these low states normal pulsations were observed. During an observation of Vela X-1 for 12 consecutive orbits in January 1998 by the Rossi X-ray Timing Explorer ( $R X T E)$, we have by chance observed such a quiescent state for the first time from the beginning to the end, preceded and followed by the usual pulsations.

\section{LIGHTCURVES AND PULSE PROFILES}

As Fig. 1 demonstrates, the source flux suddenly decreased between orbits 2 and 3, reaching its minimum during orbit 4 . At the same time the source pulsations decreased strongly, while significant non-pulsed source flux remained. This is shown in detail in Fig. 2. The pulsed fraction decreased from 30\%-50\%, depending on the energy band, to $7 \%-9 \%$. Note that even at the lowest state, the overall source flux was $>5$ times the predicted background level in the energy range used. 


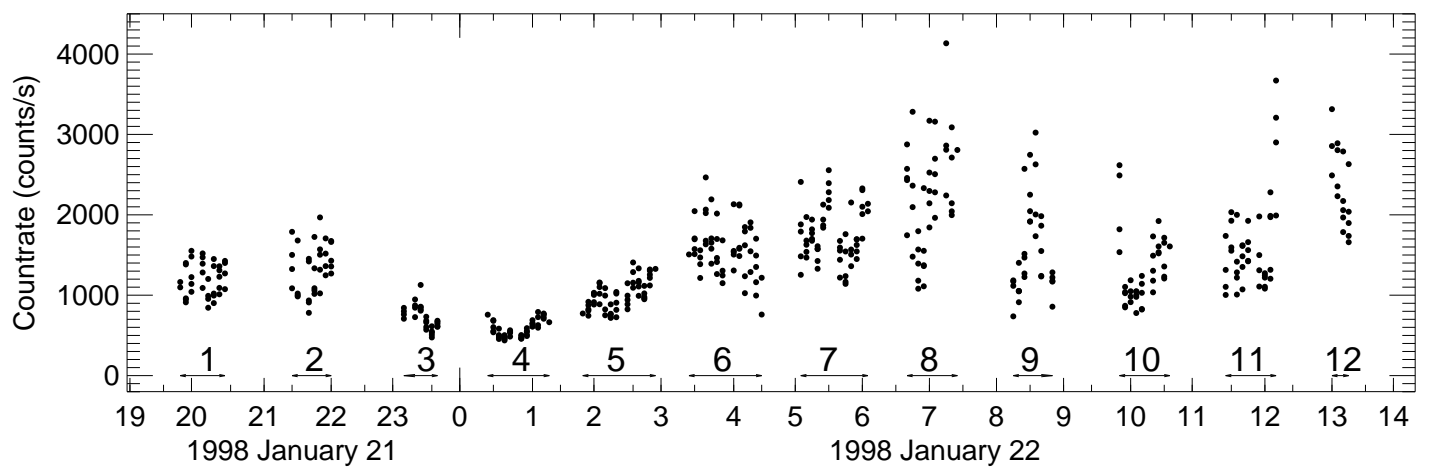

FIGURE 1. Light curve of the complete observation. The individual RXTE orbits are indicated below the light curve.
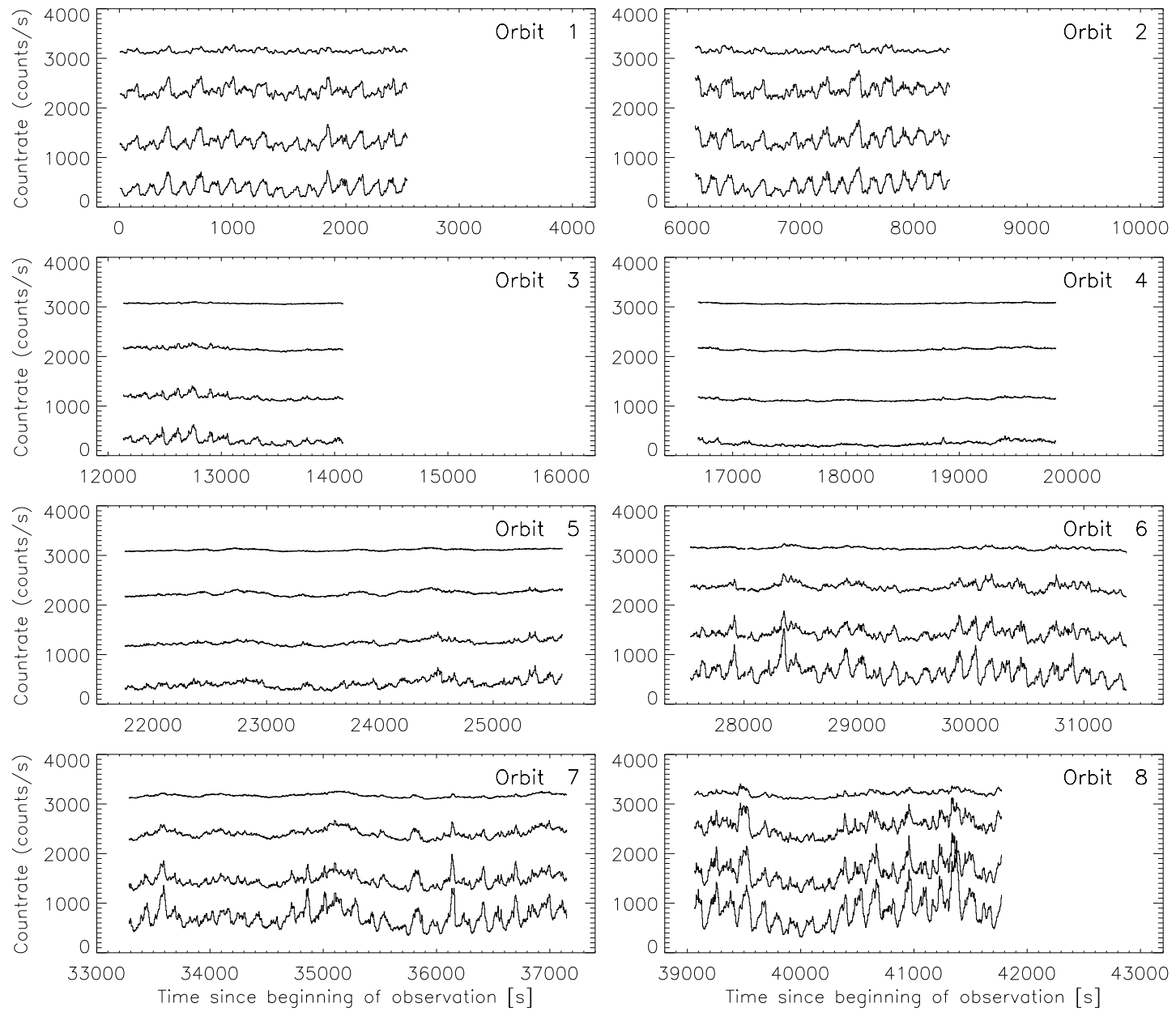

FIGURE 2. Lightcurves in four energy bands for the individual orbits 1 to 8 . The energy bands are the same as for the pulse profiles (see Fig. 3). The lightcurve for the highest energy band is plotted at the bottom with its actual count rate, the others have been shifted upwards by 1000, 2000 and 3000 counts/s respectively. 


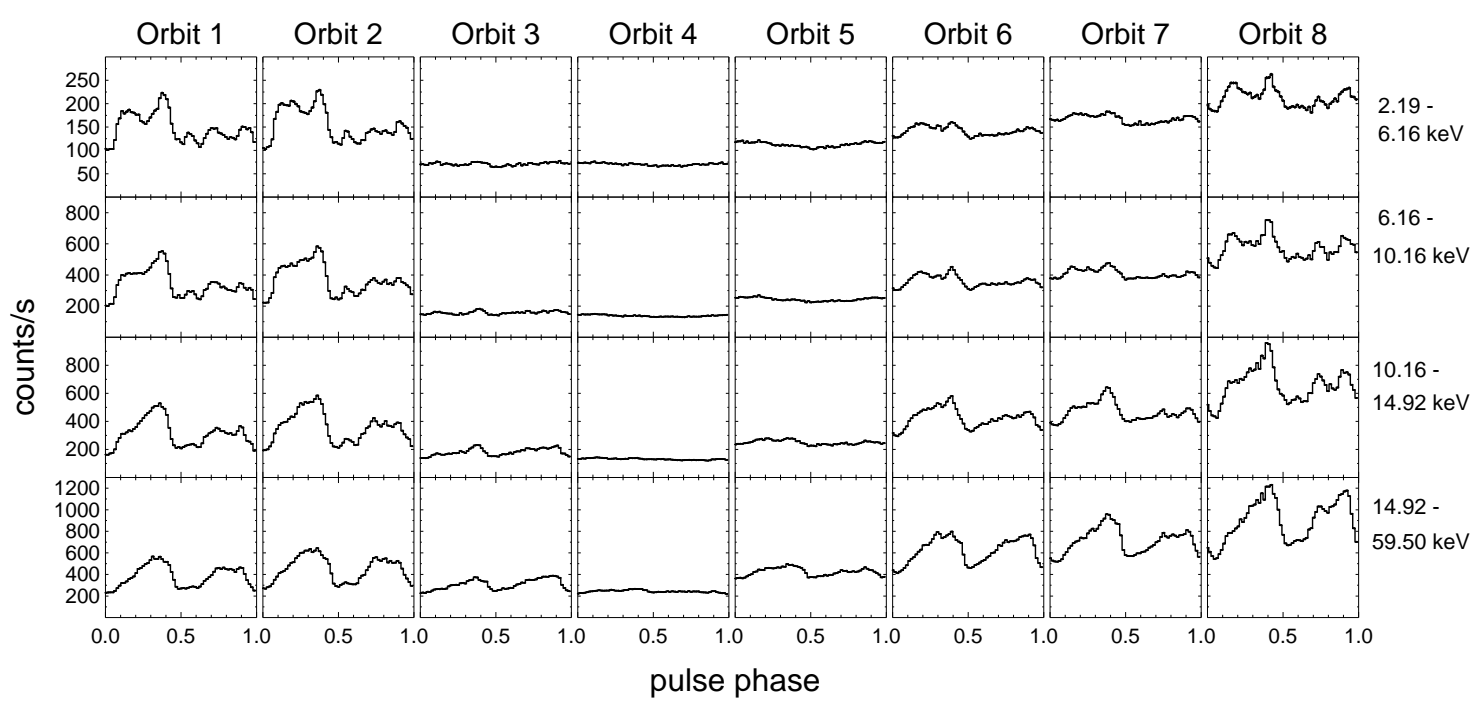

FIGURE 3. Evolution of the pulse profile for the individual orbits 1 to 8 . The energy bands are the same used in Fig. 2. The lightcurves were folded with a fixed period (283.3 s) and a common zero time.

Fig. 3 presents the pulse profiles obtained from the individual orbits 1 to 8 . The profiles of the first two orbits correspond to the well-known, complex shape usually obtained when integrating over many pulse periods, with a clear transition from a five-peaked profile at low energies to a double-peak structure at high energies (Raubenheimer, 1990). In contrast, orbits 3 to 5 show much less pronounced profiles, with the profile of orbit 4 being essentially flat. The pulse profile of the source during "recovery" (orbits $6 \&$ 7) is similar to those observed before the low state at higher energies but much less pronounced at energies $<10 \mathrm{keV}$.

\section{HARDNESS RATIOS AND SPECTRA}

Fig. 4 shows the evolution of the spectral hardness, both at energies up to $10 \mathrm{keV}$ (energy band 2 vs. band 1) and at energies beyond $10 \mathrm{keV}$ (energy band 4 vs. band 3). The hardness ratios were calculated using $(H-S) /(H+S)$ where $H$ and $S$ are the fluxes in the hard and soft band respectively. There are three apparent properties of this plot:

1. The two hardness ratios are very clearly anticorrelated.

2. The disappearence of the pulses in orbit 3 goes hand in hand with an abrupt spectral change.

3. The reemergence of pulsations is accompanied by a "normalization" of the hardness ratios, but during the high state the spectrum stays significantly harder than in the normal state at the beginning.

The strong spectral changes at the onset of the low state are also apparent in the quotient spectra shown in Fig. 5. There are clear indications for strong absorption and the same time increased flux in the iron line and a soft excess at the lowest energy range. 


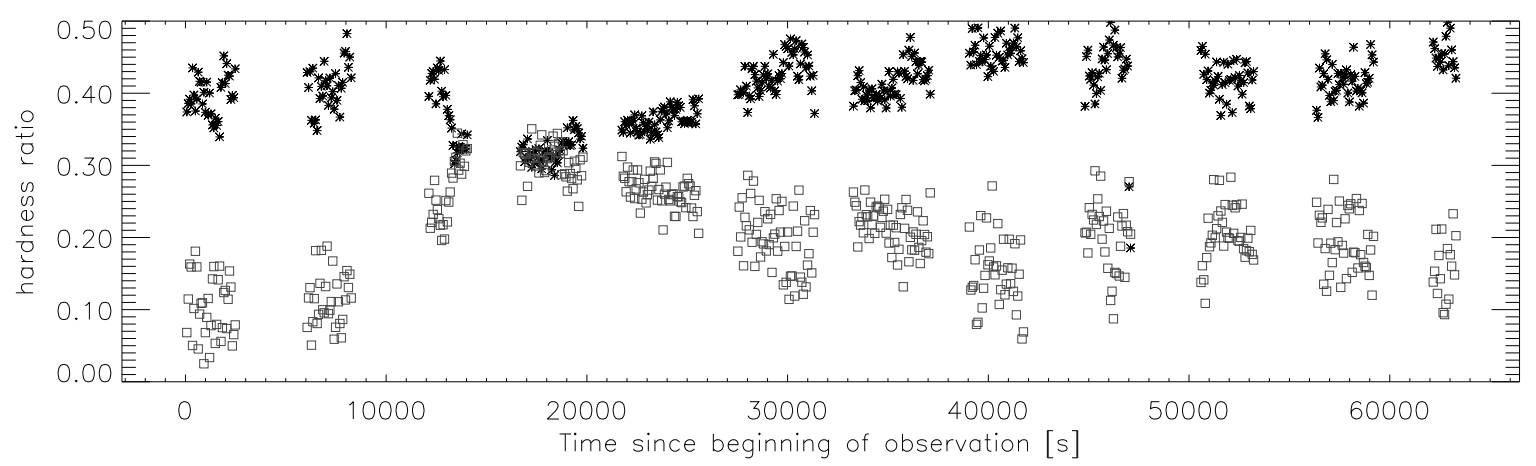

FIGURE 4. Evolution of the spectral hardness over the complete observation. The upper curve (stars) displays the ratio of energy band 2 to band 1, the lower curve (open squares) the ratio of band 4 to band 3 .
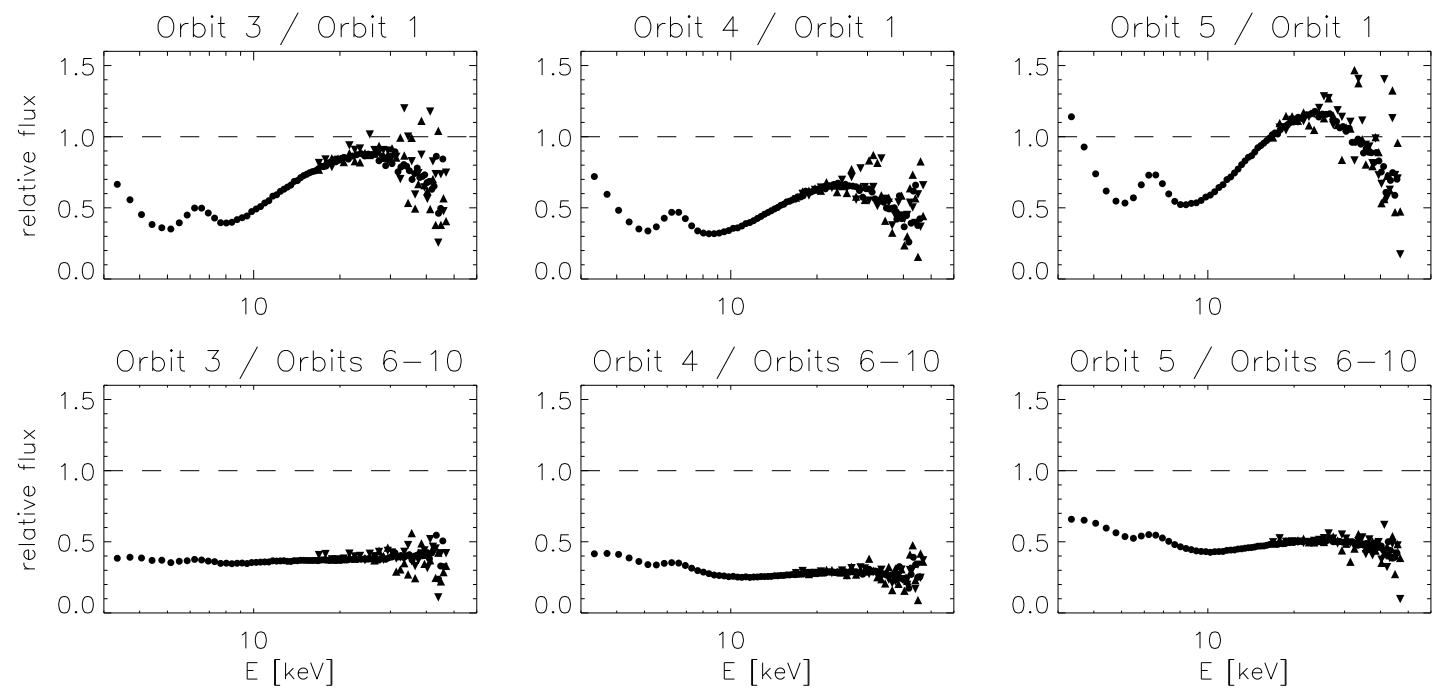

FIGURE 5. Quotient spectra of the three "low-state" orbits, with PCA data plotted as filled circles and HEXTE data as triangles: 1. upper panels: ratio to the spectrum of the normal state before the low state, 2. lower panels: ratio to the average spectrum of the active high state following the low state.

In contrast there is little change in the global spectral shape as the source begins to pulsate again. Except for a slight soft excess, the spectrum during the low state is rather well described by simply scaling the spectrum of the following flaring state.

Attempts to fit the spectra turned out to be quite difficult, even allowing for the known complexity of the Vela X-1 continuum. Detailed results have been presented on two posters at the X-ray'999 conference in Bologna, the following paragraphs summarize the results.

We used a partial covering model with two additive components, using the same continuum, one heavily absorbed and one scattered unabsorbed into the direction of the observer. The continuum used was the NPEX (Negative Positive EXponential Mihara 1995). This model had to be further modified by an additive iron line and two coupled cyclotron lines. For the high state a cyclotron line at $\sim 55 \mathrm{keV}$ is required by the data, a second feature at $\sim 21 \mathrm{keV}$ may be due to uncertainties in the PCA response matrix. 
Modeling the spectra of the individual orbits in the first part of our observation, we found the $\mathrm{N}_{\mathrm{H}}$ value of the absorbed component varying between $40 \times 10^{22} \mathrm{~cm}^{-2}$ and $230 \times 10^{22} \mathrm{~cm}^{-2}$ with a clear maximum during orbit 4 . The relative importance of the scattered component appears also to be maximal during the low state. There is no clear correlation of the other continuum parameters with source flux, but their values are quite different before and after the low state.

\section{DISCUSSION}

The results of spectral fitting are somewhat in contrast with the finding above that the global spectral shape remains more or less constant after orbit 3 . Within the framework of our spectral model this similarity is obtained by parallel changes in the column depth and in the spectral continuum. Further analysis will have to show if this is an artefact or reality.

A possible scenario to explain the disappearing pulses is that a very thick blob in the surrounding stellar wind - which is known to be clumpy (e.g., Nagase et al., 1986) - temporarily obscures the pulsar. Taking our fit results from above as basis $\left(\mathrm{N}_{\mathrm{H}, \max } \approx 2 \times 10^{24} \mathrm{~cm}^{-2}\right)$, the optical depth for Thomson scattering of such a blob would be $\sim 1.6$, reducing the direct component to $\sim 20 \%$. The scattered radiation would need to come from a relatively large region $\left(\sim 10^{13} \mathrm{~cm}\right)$ to destroy coherence. The large fraction of scattered radiation would also explain the relatively increased Fe-line emission and soft excess.

After the quiescent state, when pulsations begin again, the emission in this scenario would be a combination of heavily absorbed direct radiation - the accretion being fueled by some part of the blob - and scattered radiation from a wide region. This would also explain the reduced pulse fraction, due mainly to a higher "pedestal" during the high state as compared to the normal state at the beginning of the observation (see Fig. 3).

\section{References}

Inoue H., Ogawara Y., Ohashi T., et al., 1984, PASJ 36, 709

Kreykenbohm I., Kretschmar P., Wilms J., et al., 1999, A\&A 341, 141

Mihara T., 1995, Ph.D. thesis, University of Tokyo

Nagase F., Hayakawa S., Sato N., 1986, PASJ 38, 547

Raubenheimer B.C., 1990, A\&A 234, 172

van Kerkwijk M.H., van Paradijs J., Zuiderwijk E.J., et al., 1995, A\&A 303, 483 